\title{
Ferramentas de pedometria para caracterização da composição granulométrica de perfis de solos hidromórficos
}

\author{
Helena Saraiva Koenow Pinheiro(1), César da Silva Chagas ${ }^{(2)}$, \\ Waldir de Carvalho Júnior ${ }^{(2)}$ e Lúcia Helena Cunha dos Anjos ${ }^{(1)}$
}

\begin{abstract}
(1)Universidade Federal Rural do Rio de Janeiro, Departamento de Solos, BR 465, Km 7, CEP 23890-000 Seropédica, RJ. E-mail: lenask@gmail.com, lanjos@ufrrj.br (2)Embrapa Solos, Rua Jardim Botânico, no 1.024, Jardim Botânico, CEP 22460-000 Rio de Janeiro, RJ. E-mail: chagas.rj@gmail.com, waldir.carvalho@embrapa.br
\end{abstract}

\begin{abstract}
Resumo - O objetivo deste trabalho foi avaliar ferramentas pedométricas para caracterização da composição granulométrica (areia, silte e argila) e comparação de perfis de solos hidromórficos. O estudo foi realizado na bacia hidrográfica do rio Guapi-Macacu, RJ. Foram aplicados o algoritmo "slice-wise" para o fatiamento do perfil em intervalos de $1 \mathrm{~cm}$, e a função "spline" para harmonizar o conjunto de dados segundo seis intervalos de profundidade predefinidos. A análise do conjunto de perfis, por meio das funções de profundidade (slice-wise e spline) e de dendrograma de dissimilaridade, revelou que Planossolos e Gleissolos são relativamente rasos e apresentam camadas arenosas nos horizontes superficiais e teores de argila e silte irregulares em subsuperficie. Essas duas ordens ocorrem na área da bacia hidrográfica geralmente em planícies aluviais, em vales e em sopés de elevação. Os Neossolos Flúvicos apresentaram maiores quantidades de argila e silte em superfície, as quais decrescem em profundidade, e são mais profundos e ocorrem nos terraços aluviais. A abordagem permitiu descrever a variabilidade da textura dos solos hidromórficos em perfil e realizar a análise de similaridade entre as ordens, o que pode apoiar a distinção de fases em unidades de mapeamento e a identificação de critérios quantitativos para a classificação dos solos.
\end{abstract}

Termos para indexação: algoritmo para pedologia quantitativa, banco de dados pedológicos, classificação do solo, funções de profundidade, harmonização de dados, mapeamento do solo.

\section{Pedometric tools to characterize soil granulometric composition from hydromorphical soil profiles}

\begin{abstract}
The objective of this work was to evaluate pedometric tools to characterize the particle size distribution (sand, silt, and clay) and to compare the profiles of hydromorphic soils. The study was carried out in the Guapi-Macacu watershed, in the state of Rio de Janeiro, Brazil. The slice-wise algorithm was applied to slice the profile data at $1-\mathrm{cm}$ intervals, and the equal-area spline function for database harmonization, according to six predefined depth intervals. The analysis of the soil profile collection, using the soil-depth functions (slice-wise and spline) and the similarity analysis, revealed that Alfisols and Entisols are relatively shallow and have coarse texture in the topsoil layer and a more irregular distribution of clay and silt along the soil profile. These two orders usually occur in the watershed area related to floodplains, valleys, and footslopes. Fluvisols had higher amounts of clay and silt in the topsoil layer, which decrease with soil depth, and they are deeper and occur in alluvial terraces. The approach allowed to characterize the variability of texture along hydromorphical soil profiles and to perform a similarity analysis between soil orders, which can support the differentiation of soil mapping units and the identification of quantitative criteria for soil classification.
\end{abstract}

Index terms: algorithm for quantitative pedology, pedological database, soil classification, soil-depth functions, harmonization of soil data, soil mapping.

\section{Introdução}

Solos hidromórficos são geralmente agrupados em unidades de mapeamento que não permitem diferenciação quanto a seu potencial ou limitações de uso. Da mesma forma, o planejamento de uso e ocupação de áreas com complexos de solos hidromórficos constitui-se tarefa difícil, dada a heterogeneidade desses solos na paisagem, tanto em escala horizontal como vertical. A textura é um parâmetro-chave para diferenciar esses solos, além de também ser usada em modelos de predição para estimar parâmetros hidrológicos, densidade e estoque de carbono (Thompson et al., 2012). 
A composição da fração mineral do solo (areia, silte e argila) é um atributo altamente variável espacialmente, em profundidade -, porém de grande importância para planejamento do uso do solo. Os efeitos da textura do solo sobre a aptidão das terras e sua capacidade de armazenamento de água e nutrientes são amplamente conhecidos e estudados (Klingebiel, 1963; Jenny, 1980; Silver et al., 2000; FernandezIllescas et al., 2001).

O mapeamento da distribuição espacial dessa propriedade tem sido objetivo de estudos que incorporam geotecnologias e conceitos de pedometria, no levantamento de solos (Moore et al., 1993; Arrouays et al., 1995; McBratney et al., 2000). No entanto, no que tange à variação vertical da composição granulométrica, mais estudos são necessários para melhor entendimento desta propriedade dos solos. Nesse sentido, os esforços empregados na pedometria propõem a aplicação de funções de profundidade para representação da variabilidade de propriedades do solo em perfil, conforme demonstrado por Ponce-Hernandez et al. (1986), Bishop et al. (1999), Minasny et al. (2006), Malone et al. (2009, 2011), Mishra et al. (2009), Lacoste et al. (2014), Liu et al. (2016) e Wiese et al. (2016). A grande maioria desses estudos aplicam variações da função "spline" e tratam da avaliação do estoque de carbono orgânico em função da profundidade do solo.

No Brasil, a abordagem quantitativa para estudo de propriedades do solo em profundidade foi exemplificada no trabalho de Zinn et al. (2005) e Pádua et al. (2015), que estudaram relações entre a composição granulométrica e a densidade do solo e carbono orgânico, respectivamente. Pinheiro (2015) apresentou a aplicação de funções de profundidade para harmonização de banco de dados de perfis e posterior mapeamento dos componentes granulométricos (areia, silte e argila) na camada superficial do solo. Carvalho Junior et al. (2015) mostraram o uso da técnica de fatiamento do perfil ("slice-wise") para caracterização numérica do comportamento de propriedades como argila, areia, saturação por bases, $\mathrm{pH}$ e carbono orgânico, em função da profundidade em uma coleção de perfis.

Ao abordar a variabilidade das propriedades do solo em profundidade, o Consórcio Mundial para o Mapeamento de Solos ("GlobalSoilMap Project") propôs a harmonização de dados em profundidades, de acordo com os seguintes intervalos predefinidos: $0-5, \quad 5-15, \quad 15-30,30-60,60-100, \quad 100-200 \mathrm{~cm}$
(Hartemink et al., 2010; Arrouays et al., 2014). No caso da harmonização de dados, os intervalos predefinidos atuam como coeficientes para uma função de profundidade denominada função spline (PonceHernandez et al., 1986; Bishop et al., 1999).

Outra abordagem para estudo de coleções de perfis de solo foi apresentada por Beaudette et al. (2013), com o uso do pacote estatístico de interface no programa $\mathrm{R}$ denominado Algoritmos para Pedologia Quantitativa (AQP, “Algorithms for Quantitative Pedology"). Esse pacote apresenta um conjunto de funções para análise orientada de banco de dados de solos, como interpolação de valores de atributos do solo em perfis, comparações de similaridade/dissimilaridade e padronização da representação de horizontes e perfis de solo por meio de esboços padronizados ("sketches"). Embora seja uma abordagem interessante, do ponto de vista da análise de bancos de dados pedológicos, a aplicação dessas técnicas no Brasil é recente, e mesmo internacionalmente, é pouco difundida.

Esboço esquemático, funções de profundidade para caracterização de propriedades do solo e harmonização de dados são ferramentas que podem ser usadas para comparar coleções de perfis, na modelagem da distribuição de atributos do solo por horizontes/ camadas, e para fins taxonômicos e de mapeamento (Beaudette et al., 2013). A análise da similaridade entre perfis de solo é baseada em critérios usados para a classificação do solo, em que a comparação quantitativa deve representar a variabilidade da espessura do horizonte diagnóstico associado com as propriedades do solo (Webster \& Oliver, 1990). A hipótese empregada assume que por meio de funções de profundidade é possível representar quantitativamente o conteúdo dos componentes granulométricos em qualquer profundidade que se deseje.

O objetivo deste estudo foi avaliar ferramentas pedométricas para caracterização da composição granulométrica (areia, silte e argila) e comparação de perfis de solos hidromórficos da bacia hidrográfica do rio Guapi-Macacu, no Estado do Rio de Janeiro.

\section{Material e Métodos}

A bacia hidrográfica do rio Guapi-Macacu possui área de contribuição de $1.250,78 \mathrm{~km}^{2}$ e perímetro de 199,2 km, e abrange o município de Cachoeiras de Macacu e partes de Itaboraí e de Guapimirim, na Região

Pesq. agropec. bras., Brasília, v.51, n.9, p.1326-1338, set. 2016 DOI: $10.1590 / \mathrm{S} 0100-204 \mathrm{X} 2016000900032$ 
Hidrográfica da Baía de Guanabara, no Estado do Rio de Janeiro (Figura 1). A região está localizada na parte central do Gráben da Guanabara, classificada como Bacia Sedimentar Macacu, formada por várias sequências de deposição de eventos tectônicos no início do Terciário (Ferrari, 2001). De acordo com Projeto Macacu (2010), o clima é Aw (tropical chuvoso, com inverno seco), segundo Köppen (1948), com temperatura média anual de $23^{\circ} \mathrm{C}$ e precipitação média anual entre $1.200 \mathrm{~mm}$ e $2.600 \mathrm{~mm}$ (contrafortes serranos). A região faz parte do bioma Mata Atlântica e apresenta diferentes tipos de vegetação natural de acordo com peculiaridades da paisagem tais como campos de altitude, florestas densas, manguezais, pântanos e estuários (Pedreira et al., 2009).

O levantamento pedológico foi realizado entre 2011 e 2012, com coleta de cerca de cem perfis nas diferentes feições da paisagem e na totalidade da bacia do rio Guapi-Macacu. Os pontos amostrais foram selecionados pelo algoritmo "Conditioned Latin Hipercube Sampling" (cLHS) (Minasny \& McBratney, 2006; Roudier et al., 2012), visando efeito aleatório e a melhor distribuição da variação das características da paisagem, considerando ainda a viabilidade de acesso por meio da restrição pela distância em relação à malha viária (predefinida em $100 \mathrm{~m}$ em cada lado da estrada). Detalhes sobre o procedimento estão descritos em Pinheiro et al. (2014). O mapa de Uso do Solo foi adaptado de Fidalgo et al. (2008) e teve importante papel na seleção do conjunto amostral, para restrição da seleção de pontos em áreas urbanizadas.

A descrição dos perfis e a coleta de amostras para determinar os atributos do solo foi realizada

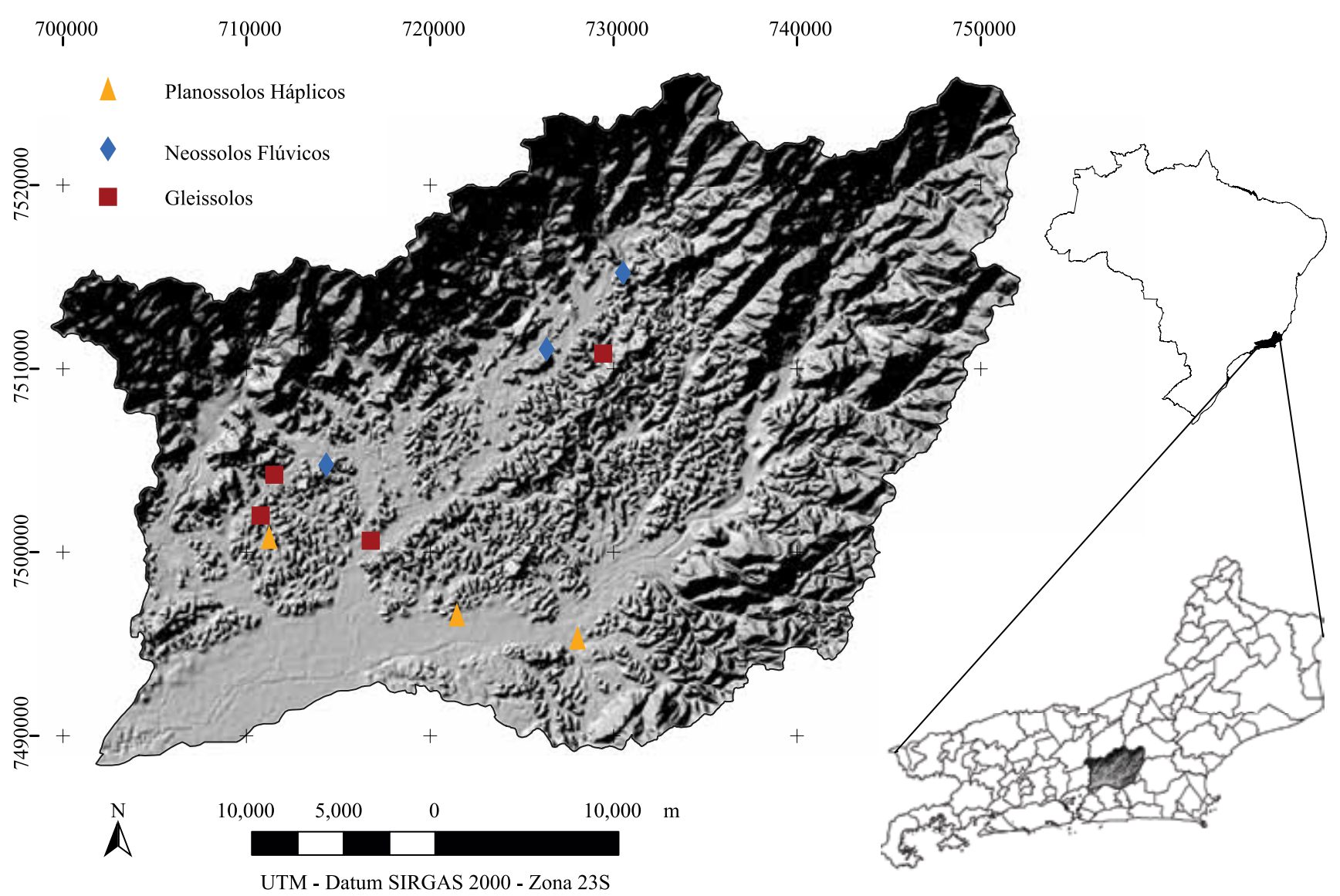

Figura 1. Localização da bacia hidrográfica do rio Guapi-Macacu, RJ, e do conjunto de perfis de solos hidromórficos selecionados (10 perfis), composto por quatro perfis de Gleissolos (vermelho), três de Planossolos Háplico (laranja) e três de Neossolos Flúvicos (azul). 
de acordo com Santos et al. (2005) e os solos foram classificados até o quarto nível categórico, segundo o Sistema Brasileiro de Classificação de Solos (SiBCS) (Santos et al., 2013). As análises de rotina foram feitas conforme Claessen (1997) e foram selecionados dados de cor do solo (úmida), areia total, silte e argila. O conjunto de dados é composto de dez perfis de solos (coleção) com dados analíticos e descrição completa dos horizontes, sendo quatro Gleissolos (Háplicos e Melânicos), três Neossolos Flúvicos e três Planossolos Háplicos. A Figura 1 mostra a localização da bacia hidrográfica do rio Guapi-Macacu, RJ, e dos dez perfis de solos hidromórficos selecionados para uso no presente estudo, também referido como "coleção".

Foram criados modelos numéricos para descrever a variabilidade do tamanho das partículas do solo (areia, silte e argila) em profundidade. A análise compreendeu a aplicação dos algoritmos "slice-wise" e "equal-area spline function" para a estimativa dos dados em intervalos regulares $(1 \mathrm{~cm})$ e em seis intervalos predefinidos de acordo com a proposta do Consórcio Mundial para o Mapeamento de Solos (camadas de $0-5,5-15,15-30,30-60,60-100,100-200 \mathrm{~cm})$, respectivamente.

Os procedimentos estatísticos para analisar os dados e implementar as funções de profundidade do solo foram feitos no programa de livre acesso $\mathrm{R}$ (The R Foundation, 2013). Para plotar as funções de profundidade do solo foi usado o pacote Algoritmos para Pedologia Quantitativa (AQP, Algorithm for Quantitative Pedology), desenvolvido por Beaudette et al. (2013). Para adequar as especificações do "GlobalSoilMap Project", a partir da base original foi gerada base de dados harmonizada em seis profundidades predefinidas (camadas de $0-5,5-15$, 15-30, 30-60, 60-100, 100-200 cm). Na harmonização dos dados foi aplicada a função "spline" que usa algoritmo interpolador para estimar os valores médios relativos a cada uma das seis camadas. A função spline, proposta por Ponce-Hernandez et al. (1986), representa função não paramétrica, designada como "equal-area spline function", que é apropriada para modelagem de atributos do solo (Bishop et al., 1999; Malone et al., 2009). Essa função ajusta os dados para cada camada de espessura predefinida, de forma que os nós que intersectam cada camada estejam perto da inflexão da curva de ajuste e o mais longe possível das bordas da camada, preservando o valor médio da propriedade do solo (Odgers et al., 2012).

A análise dos dados de textura dos perfis compreendeu também a aplicação do algoritmo slice-wise (Beaudette et al., 2013), que possibilita o "fatiamento" do solo, atribuindo valores para cada intervalo de $1 \mathrm{~cm}$. Este artifício matemático possibilita, a partir de uma base de dados, a computação estatística de valores ponderados para segmentos, reconstruindo os dados do perfil em uma nova base de acordo com profundidades predefinidas, representando uma 'função de profundidade do solo', também referida na literatura como "soil-depth function" (Beaudette et al., 2013). A aplicação do algoritmo slice-wise adota a premissa de que uma função representativa para a variação de propriedades do solo em profundidade pode ser gerada a partir de uma coleção de dados de perfis, sumarizados em intervalos de profundidade (Beaudette et al., 2013). O fatiamento permite o cálculo dos valores médios e medianos para cada segmento por meio de vetor calculado de acordo com a sintaxe abaixo: \{slab (dados $\sim$ argila + areia + silte, slab.fun $=$ media/mediana, na.rm=True) $\}$ - Linha de comando.

A partir da base de dados gerada para camadas de $1 \mathrm{~cm}$ de espessura, foi calculada a matriz de dissimilaridade entre os perfis, para cada camada, permitindo avaliar a similaridade. A dissimilaridade entre os solos hidromórficos foi calculada usando a função "profile compare", tendo como base para a comparação o conteúdo de areia, argila, e silte, considerando a profundidade máxima e coeficiente de ponderação igual a 0,01 . O número de perfis utilizado no cálculo é representado pela fração de contribuição, cujo valor pode ser considerado como medida agregada de probabilidade para cada profundidade do solo. Adicionalmente, foi usada uma função para renderizar os esquemas dos perfis, de acordo com a carta de cores Munsell para solos. O algoritmo não comporta a representação de mosqueados e colorações variegadas, típicas de solos hidromórficos. Ainda assim, é útil para padronizar a visualização de esboços de perfis de solo, permitindo análises e comparações para fins taxonômicos e/ou utilitários.

O fluxograma na Figura 2 ilustra a metodologia descrita e apresenta a sequência dos procedimentos realizados.

Pesq. agropec. bras., Brasília, v.51, n.9, p.1326-1338, set. 2016 DOI: $10.1590 / \mathrm{S} 0100-204 \mathrm{X} 2016000900032$ 


\section{Resultados e Discussão}

$\mathrm{Na}$ bacia hidrográfica do rio Guapi-Macacu, as planícies fluviais ocupam cerca de $20 \%$ da área e os solos hidromórficos são encontrados sobre depósitos de sedimentos quaternários, geralmente com elevação inferior a $20 \mathrm{~m}$ do nível do mar, declividade inferior a $3 \%$ e curvatura planar $(-0,01$ a 0,001$)$, com predomínio das classes: Planossolos (Háplicos e Argilúvicos), Gleissolos (Háplicos e Melânicos), Neossolos (Flúvicos e Quartzarênicos). A diferença taxonômica entre as classes consiste basicamente na expressão do horizonte glei, nos Gleissolos, ou horizonte plânico, nos Planossolos, ou ausência de expressão de processos pedogenéticos e presença de caráter flúvico, nos Neossolos Flúvicos. A Tabela 1 apresenta os dados descritivos dos perfis selecionados.

Os menores valores para fração argila e silte foram observados nos perfis de Neossolos Flúvicos (40 e $29 \mathrm{~g} \mathrm{~kg}^{-1}$, respectivamente), assim como os maiores teores de areia total (maiores que 90\%). No entanto, os Planossolos Háplicos apresentaram medidas de tendência central (media e mediana) com maiores valores para areia total $\left(755,5\right.$ e $768,0 \mathrm{~g} \mathrm{~kg}^{-1}$, respectivamente). Os perfis de solos hidromórficos apresentam, de uma forma geral, textura arenosa com valores médios dos componentes silte e argila em torno de $160 \mathrm{~g} \mathrm{~kg}^{-1}$, e de $680 \mathrm{~g} \mathrm{~kg}^{-1}$ para areia total. Porém, observa-se grande variabilidade, considerando a perspectiva em profundidade, o que justifica o uso de outras ferramentas de análise para melhor caracterização e comparação da composição granulométrica desses perfis. A Figura 3 ilustra o conteúdo de areia, silte e argila por horizonte e na perspectiva dos perfis de solo. As cores atribuídas aos horizontes representam o conteúdo dos componentes da composição granulométrica em intervalos de $200 \mathrm{~g} \mathrm{~kg}^{-1}$ para areia (Figura 3) e $100 \mathrm{~g} \mathrm{~kg}^{-1}$ para os demais componentes.

Esboços esquemáticos para representação de perfis de solo são úteis para os pedólogos, uma vez que retratam a estratigrafia de horizontes e suas transições (Beaudette et al., 2013). No entanto, esses esboços não estão em escala e não têm legendas ou simbologia padrão; assim, são subjetivos e dificultam a transmissão da informação para outros usuários.

A visualização em esboços padronizados, sketches, de acordo com Beaudette et al. (2013), permitiu destacar predominâncias na composição granulométrica dos horizontes das distintas ordens dos solos hidromórficos (Figura 3). Os Planossolos apresentam valores superiores para areia, em superfície, e argila no horizonte subsuperficial, o que condiz com os critérios diagnósticos para esta ordem. Os Neossolos Flúvicos, por sua vez, apresentam teores

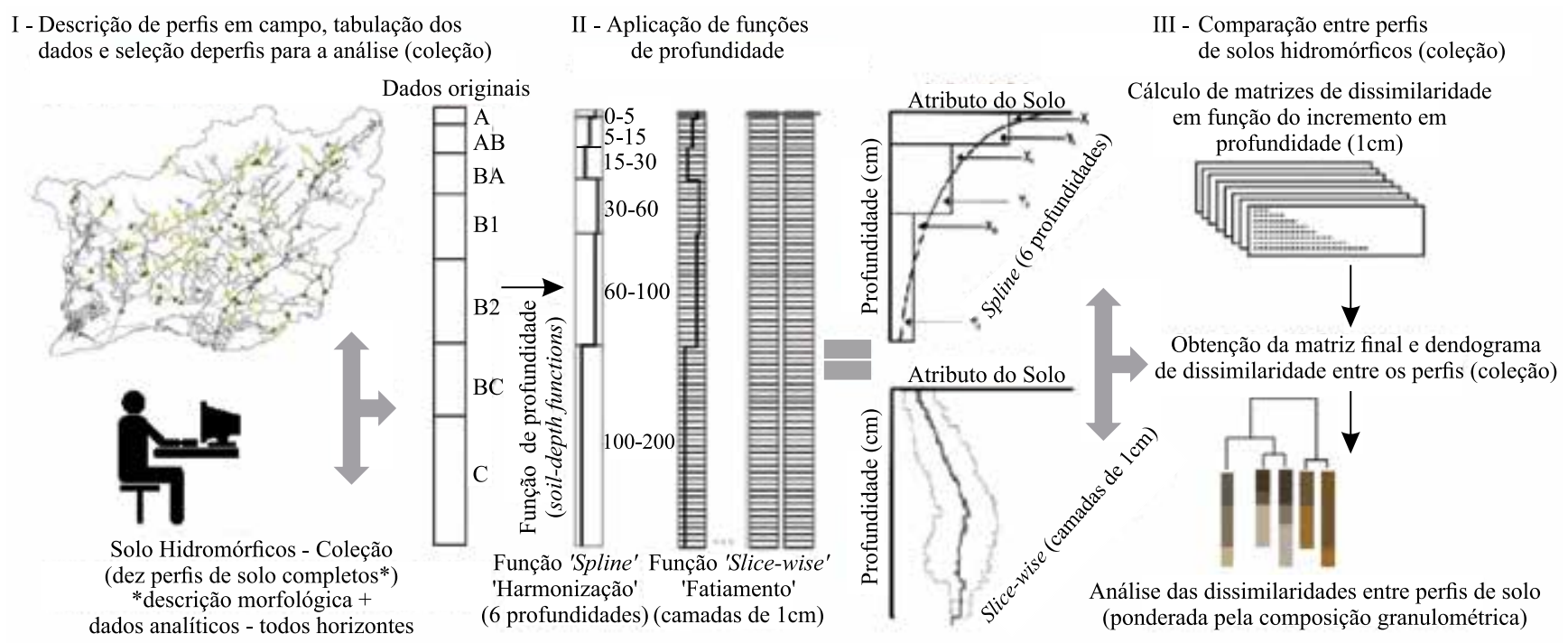

Figura 2. Fluxograma metodológico: I, coleta e descrição de perfis em campo, e tabulação e organização de banco de dados (por horizonte); II, aplicação de funções de profundidade, com fatiamento de $1 \mathrm{~cm}$ e harmonização em seis camadas; e III, comparação e análise da similaridade entre os perfis da coleção de solos hidromórficos. 
de silte relativamente mais elevados em superfície, e areia total em subsuperfície, embora a textura tenha grande variabilidade nesses solos. Os Gleissolos geralmente apresentam valores de argila crescentes em profundidade, porém não suficientes para designar gradiente textural. De forma geral, os Gleissolos apresentam semelhante padrão dos componentes da textura do solo, com exceção do perfil 14, que mostrou grande variabilidade na composição granulométrica dos distintos horizontes, conforme observado na Figura 3.

É sabido que a variação vertical de propriedades do solo tende a ser contínua em um perfil de solo e por essa razão funções de profundidade de solo são usualmente empregadas para representar esta perspectiva do perfil (Ponce-Hernandez et al., 1986; Malone et al., 2011). Dessa forma, a variação contínua na composição granulométrica de um perfil, ou coleção de perfis, pode ser mais bem observada por meio da aplicação de funções de profundidade. A Figura 4 mostra as funções para representação composição granulométrica (areia, silte e argila) em profundidade usando o algoritmo slice-wise e a função spline, em intervalos de $1 \mathrm{~cm}$ e de acordo com as profundidades sugeridas pelo Consórcio Global para o Mapeamento de Solos, respectivamente. O número de perfis que contribuem para o cálculo de cada intervalo de profundidade é descrito à direita de cada gráfico.

A agregação em profundidades predefinidas apresentou tendência similar ao fatiamento centimétrico (Figura 4), embora em profundidades maiores que $60 \mathrm{~cm}$ a função spline para agregação em seis profundidades tenha generalizado a média dos valores para as camadas $60-100$ e 100-200 cm. Nesse sentido, a variabilidade vertical contínua foi mais bem observada na Figura 4A, com advento do fatiamento em camadas de 1 centímetro (slice-wise), principalmente para profundidades entre $60-80$ e $80-100 \mathrm{~cm}$. Cabe destacar que, embora as funções de profundidade do solo representem a tendência central de uma coleção de perfis, a generalização desses dados deve ser cuidadosamente avaliada, uma vez que a contribuição de dados na análise (\% de perfis da coleção utilizados no cálculo para cada profundidade) é variável para cada espessura estimada pelas funções. Malone et al. (2009) e Minasny et al. (2006) observaram que a predição de propriedades contínuas do solo geralmente tem melhor desempenho na camada superficial do que em subsuperfície.

Por meio das funções de profundidade aplicadas para caracterização da composição granulométrica, foi possível observar que os horizontes/camadas superficiais apresentam maior variabilidade, assim como, maior número de perfis foi utilizado no cálculo (valores percentuais), conforme apresentado na Figura 4. A variabilidade na estimativa dos atributos pelas duas funções de profundidade revelou similaridade quanto à distribuição entre 25 e 75 percentis, embora os dados harmonizados não mostrem certos picos de variação dos dados, principalmente em subsuperfície a partir de $1 \mathrm{~m}$ de profundidade diante da agregação em camadas predefinidas. Portanto, devese evitar extrapolar dados para a camada de 1 a $2 \mathrm{~m}$, principalmente diante da contribuição reduzida pelo menor número de perfis no cálculo, pois somente $10 \%$ da coleção possui valores para camadas abaixo de $120 \mathrm{~cm}$ (Figura 4).

A Figura 5 apresenta a distribuição vertical das frações granulométricas para cada ordem de solo, de acordo com o fatiamento em camadas de $1 \mathrm{~cm} \mathrm{e}$ harmonizados em seis camadas de profundidades predefinidas. O comportamento da curva da função que representa a variabilidade da areia total é de forma geral inverso ao da curva que representa o conteúdo

Tabela 1. Dados descritivos de uma coleção( ${ }^{(1)}$ de perfis de solos hidromórficos.

\begin{tabular}{|c|c|c|c|c|c|c|c|c|c|c|c|c|}
\hline \multirow[t]{3}{*}{ Parâmetro } & \multicolumn{3}{|c|}{ Coleção } & \multicolumn{3}{|c|}{ Gleissolos } & \multicolumn{3}{|c|}{ Neossolos Flúvicos } & \multicolumn{3}{|c|}{ Planossolos } \\
\hline & Argila & Areia & Silte & Argila & Areia & Silte & Argila & Areia & Silte & Argila & Areia & Silte \\
\hline & \multicolumn{3}{|c|}{ 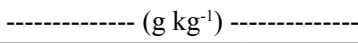 } & \multicolumn{3}{|c|}{------------ $\left(\mathrm{g} \mathrm{kg}^{-1}\right)$----------- } & \multicolumn{3}{|c|}{------------ $\left(\mathrm{g} \mathrm{kg}^{-1}\right)$----------- } & \multicolumn{3}{|c|}{ 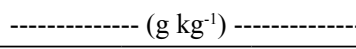 } \\
\hline Mínimo & 40,0 & 96,0 & 29,0 & 80,0 & 96,0 & 51,0 & 40,0 & 370,0 & 29,0 & 60,0 & 589,0 & 34,0 \\
\hline Média & 159,6 & 680,0 & 159,8 & 205,1 & 629,9 & 164,9 & 131,7 & 672,4 & 195,9 & 137,3 & 755,5 & 107,2 \\
\hline Mediana & 141,0 & 711,0 & 129,0 & 141,5 & 730,0 & 127,5 & 142,0 & 636,0 & 214,5 & 121,0 & 768,0 & 82,0 \\
\hline Máximo & 519,0 & 931,0 & 385,0 & 519,0 & 869,0 & 385,0 & 265,0 & 931,0 & 365,0 & 242,0 & 906,0 & 270,0 \\
\hline
\end{tabular}

${ }^{(1)}$ Coleção formada por dez perfis: quatro de Gleissolos, três de Neossolos Flúvicos e três de Planossolos. 


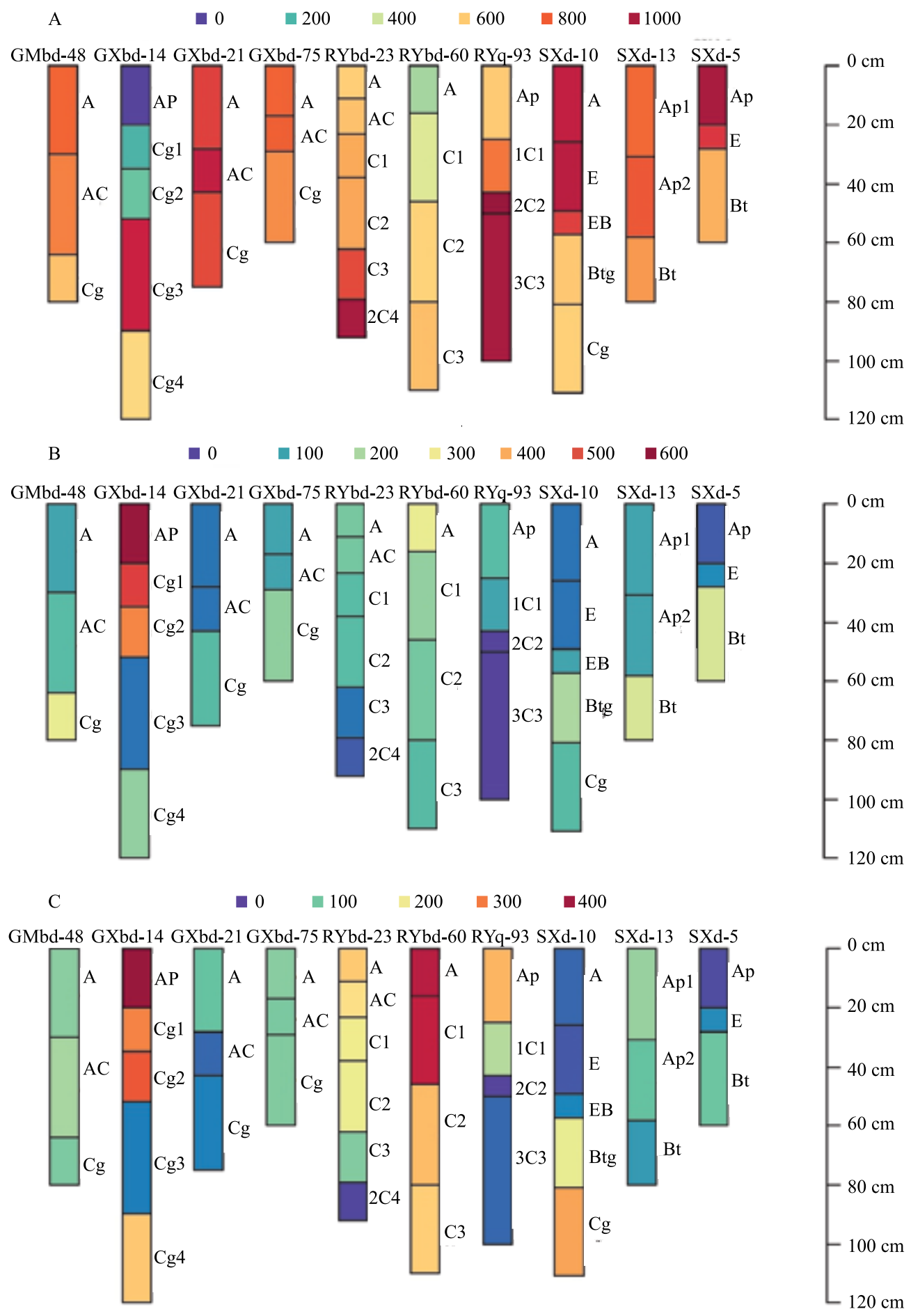

Figura 3. Composição das frações granulométricas dos horizontes de dez perfis de solos hidromórficos. A coloração representa o conteúdo da fração granulométrica, com valores expressos em $\mathrm{g} \mathrm{kg}^{-1}$, de acordo com intervalos predefinidos: A, fração areia total; B, fração argila; e C, fração silte. GMbd, Gleissolo Melânico Tb distrófico; GXbd, Gleissolo Háplico Tb distrófico; RYbd, Neossolo Flúvico Tb distrófico; RYq, Neossolo Flúvico psamítico; e SXd, Planossolo Háplico distrófico. 

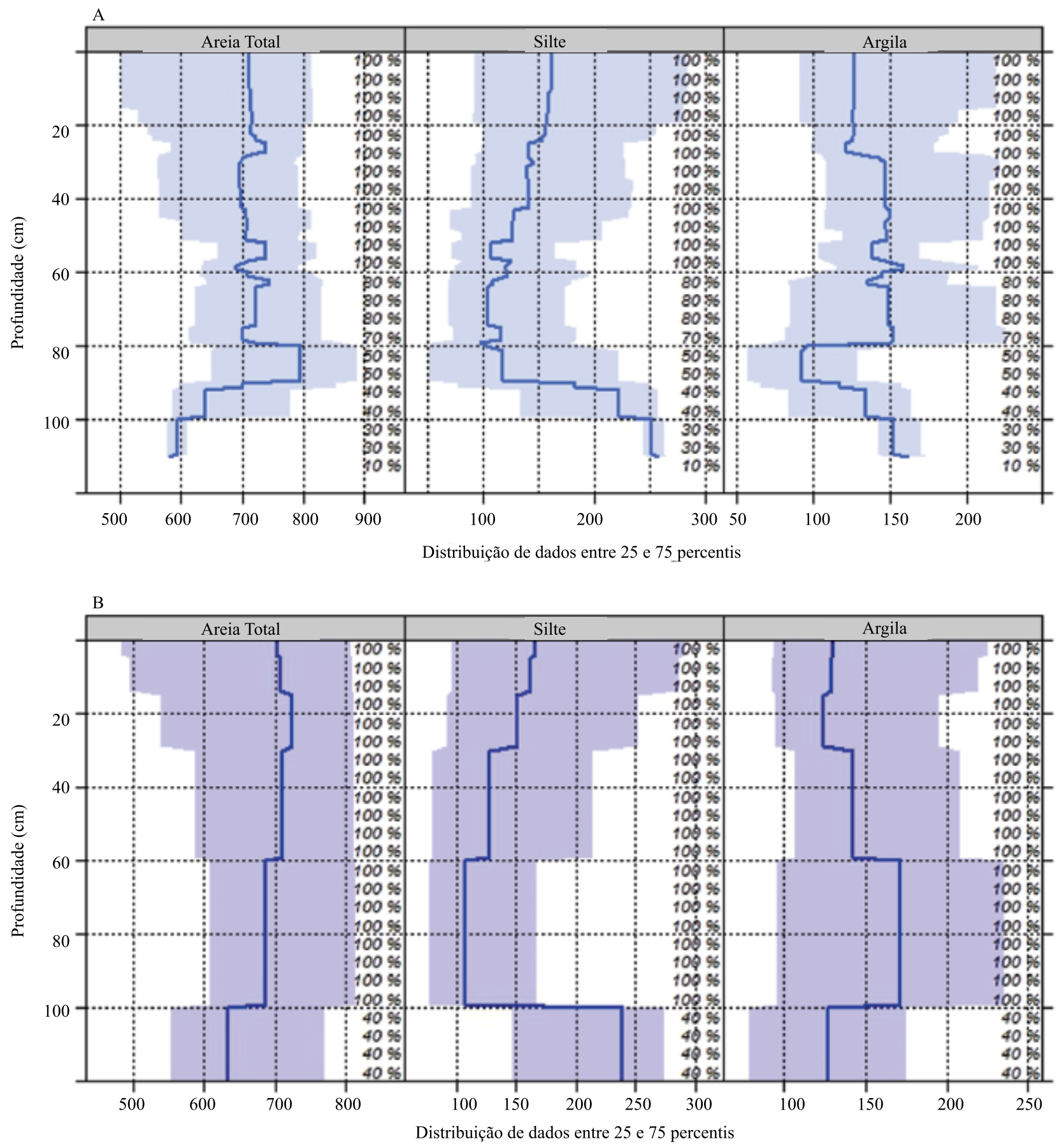

Figura 4. Distribuição do conteúdo de areia, silte, argila para a coleção de dez perfis selecionados: A, distribuição dos dados a partir do fatiamento em $1 \mathrm{~cm}$; B, distribuição de dados harmonizados em seis camadas $(0-5 ; 5-15 ; 15-30 ; 30-60 ; 60-100$; 100-200 cm). Valores expressos em $\mathrm{g} \mathrm{kg}^{-1}$. Os valores percentuais representam o número relativo de perfis que contribuiu para as estimativas em cada camada. 
de argila, principalmente quando ocorrem picos nas curvas (provavelmente relacionados às transições entre horizontes). Quanto aos teores de silte, é possível observar certa constância de valores até $50 \mathrm{~cm}$ de profundidade, seguida de pequena alteração no conteúdo, o que pode ser relacionado à presença dos horizontes diagnósticos, no caso dos Planossolos e Gleissolos.

De forma geral, os Neossolos Flúvicos apresentam redução dos teores de silte e argila com a profundidade, embora o conteúdo desses
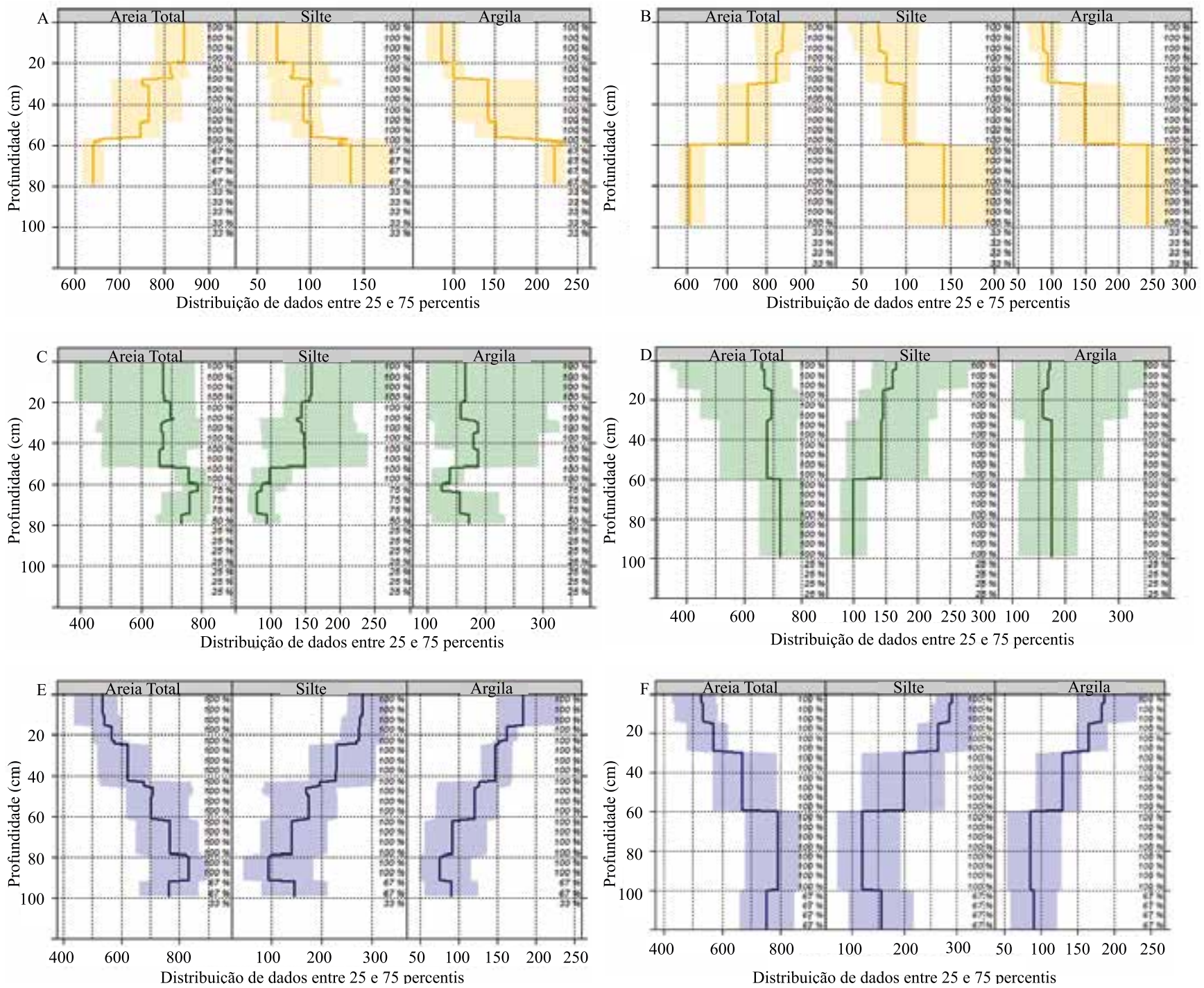

Figura 5. Distribuição do conteúdo de areia, silte, argila nas ordens de solo avaliadas: A, Planossolos Háplicos, com fatiamento dos dados em $1 \mathrm{~cm}$; B, Planossolos Háplicos, com dados harmonizados em seis profundidades; C, Gleissolos com fatiamento dos dados em $1 \mathrm{~cm}$; D, Gleissolos com dados harmonizados em seis profundidades; E, Neossolos Flúvicos com fatiamento dos dados em $1 \mathrm{~cm}$; e F, Neossolos Flúvicos com dados harmonizados em seis profundidades. Valores expressos em $\mathrm{g} \mathrm{kg}^{-1}$. Os valores percentuais representam o número relativo de perfis que contribui para as estimativas para cada profundidade.

Pesq. agropec. bras., Brasília, v.51, n.9, p.1326-1338, set. 2016

DOI: 10.1590/S0100-204X2016000900032 
positiva com o teor de areia e densidade do solo a partir de $20 \mathrm{~cm}$.

O emprego de ferramentas pedométricas para quantificar e caracterizar a composição granulométrica em profundidade possibilitou identificar diferenças entre as ordens de solos hidromórficos. As funções de profundidade para os Planossolos (Figura 5) destacam incremento de argila e silte (em menor expressão) em profundidade, particularmente após $60 \mathrm{~cm}$. Diante do conjunto de perfis coletados com mais de $1 \mathrm{~m}$ de profundidade para esta ordem (cerca de 33\%, apenas 1 perfil), não devem ser estimados valores para profundidades além de um metro. O mesmo acontece nos Gleissolos (Figura 5), em sua maioria com perfis relativamente rasos (menor que $1 \mathrm{~m}$ ) e influenciados pelo lençol freático.

Assim, grande parte dos trabalhos propostos por Zinn et al. (2005), Malone et al. (2009, 2011), Lacoste et al. (2014), Wiese et al. (2016) utilizam funções para predição de propriedades do solo até cerca de $1 \mathrm{~m}$ de profundidade, em estudos focando estoques de carbono. Já no que se refere aos objetivos deste estudo (caracterização da composição granulométrica e diferenciação entre solos hidromórficos), é justificável que a análise considere dados para profundidade superiores à camada de $1 \mathrm{~m}$ de solo, quando disponíveis.

Nos Gleissolos a variabilidade da composição textural é maior, e de forma contrária aos Planossolos, com aumento do conteúdo de areia em profundidades maiores que $50 \mathrm{~cm}$. Apesar da variabilidade da composição desses solos observada por meio da distribuição de dados entre 25 e 75 percentis, quanto aos teores de argila e silte, é possível observar que os valores médios representados pelas funções de profundidade tendem a decrescer entre a camada de $50 \mathrm{~cm}$ a $70 \mathrm{~cm}$ (Figura 5). A harmonização dos dados conforme sugerido pelo GlobalSoilMap Project, que representa os valores médios para composição da textura em intervalos profundidades predefinidos, mostra comportamento quase constante para argila nos horizontes subsuperficiais (a partir de cerca de $30 \mathrm{~cm}$ ), conforme observado na Figura 5.

O padrão para as duas funções de profundidade nos Neossolos Flúvicos foi relativamente similar (Figura 5), sugerindo boa aplicação da função spline na harmonização dos dados em intervalos predefinidos, representando a tendência média de forma mais grosseira, porém mais próxima da distribuição em intervalos de $1 \mathrm{~cm}$ (fatiamento). Nesses solos, as camadas que englobam os dados entre $30 \mathrm{~cm}$ e $60 \mathrm{~cm}$, e entre $60 \mathrm{~cm}$ e $100 \mathrm{~cm}$ apresentam na função spline uma generalização do decréscimo no conteúdo de silte e argila; e acréscimos no conteúdo de areia. Esses solos ocorrem preferencialmente em terraços e são constituídos de areias finas estratificadas e intercaladas com lentes de argila estreitas e irregulares, geralmente capeadas por alúvios mais recentes.

Nocasodos solos hidromórficos dabaciahidrográfica, a aplicação de funções de profundidade para caracterização numérica da composição granulométrica mostrou ser útil para a descrição do comportamento das variáveis areia, silte e argila, permitindo detectar mudanças em profundidade que muitas vezes não são percebidas a partir da análise os valores médios para representação de camadas predefinidas, como sugerido pelo Consórcio Mundial para o Mapeamento de Solos (GlobalSoilMap), ou horizontes genéticos, conforme coletado em campo. No entanto, mais pesquisas são necessárias uma vez que a utilização dessas técnicas para estudos de propriedades dos solos é recente, e por isso existem poucos trabalhos na literatura para análises comparativas, principalmente aplicadas às condições de solos tropicais e subtropicais.

Ferramentas pedométricas para análise de similaridade também podem ser aplicadas para comparação entre perfis de solo a partir do fatiamento com o algoritmo slice-wise que permite a criação de matrizes de dissimilaridade por profundidade, conforme proposto por Beaudette et al. (2013). A comparação de distintas ordens de solos através da análise de similaridade entre os perfis é baseada no agrupamento hierárquico divisório, assumindo que uma ordem de solo representa uma grande coleção de perfis que podem ser divididos em grupos menores e mais homogêneos, considerando características como o arranjo e propriedades morfológicas de horizontes/camadas (Kaufman \& Rousseeuw, 2005; Beaudette et al., 2013). Nesse sentido, dendrograma de dissimilaridade e esboços esquemáticos dos perfis foram criados para auxiliar a comparação entre as três ordens (Planossolos, Gleissolos e Neossolos). O eixo horizontal $(\mathrm{X})$ da Figura 6 organiza as diferenças entre pares de perfis com base no teor de argila, areia e silte; enquanto a componente vertical (Y) representa as profundidades dos horizontes de acordo com a descrição de campo. A escala do dendrograma no lado esquerdo superior da Figura corresponde à dissimilaridade

Pesq. agropec. bras., Brasília, v.51, n.9, p.1326-1338, set. 2016 DOI: $10.1590 / \mathrm{S} 0100-204 \mathrm{X} 2016000900032$ 
numérica em termos relativos (percentuais), com base na composição de areia, silte e argila.

No que tange à textura, os Gleissolos e Planossolos apresentam maior similaridade entre si, principalmente os perfis 75, 5, 48, 13. Apenas os perfis 14 (Gleissolo) e 10 (Planossolo) mostram diferenças para os demais perfis das respectivas classes (superiores a 60\%), o que pode indicar relações particulares na paisagem ou características de determinada fase de unidade de mapeamento. Os Neossolos Flúvicos mostram maior semelhança entre si, diferindo para profundidade e características texturais devido à descontinuidade litológica em alguns perfis (23 e 93). Conforme destacado por Beaudette et al. (2013), a profundidade total do horizonte em função dos dados coletados em campo influencia o cálculo da dissimilaridade entre perfis. Isso pode ser observado no dendrograma diante do agrupamento de solos, por vezes, taxonomicamente distintos, como, por exemplo, os perfis 75 e 5 e os perfis 60 e 10 (Figura 6).

Por meio dos esboços padronizados e renderizados, conforme carta Munsell de cores para solos, foi possível observar a evidência de gleização em um dos Planossolos e, conforme esperado, nos quatro Gleissolos (Figura 6). O horizonte glei ocorre entre $20 \mathrm{~cm}$ e $60 \mathrm{~cm}$ com mosqueados em muitos casos, refletindo a drenagem deficiente. Os Planossolos apresentam horizonte subsuperficial com notória

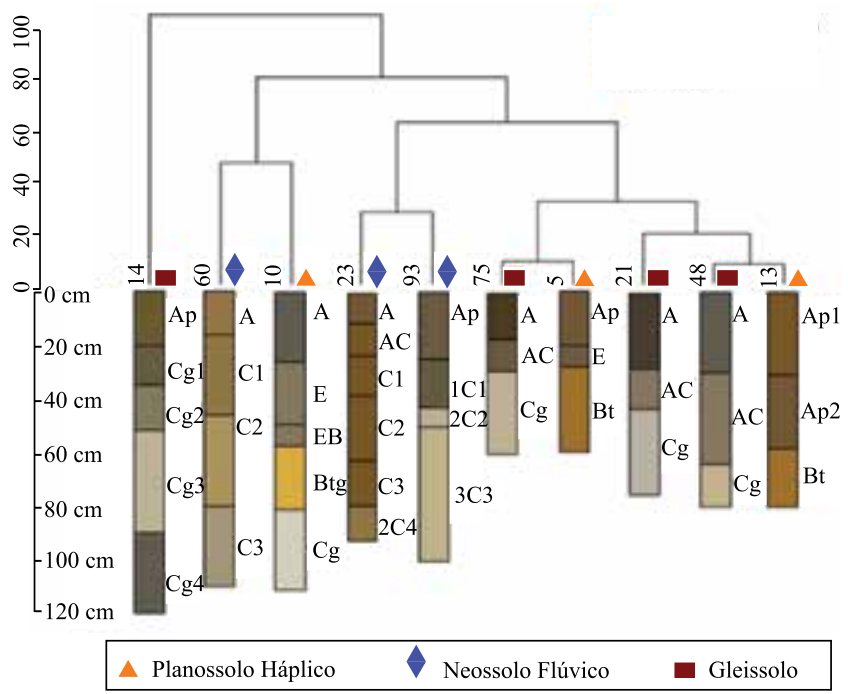

Figura 6. Dissimilaridade entre perfis de solos hidromórficos da bacia hidrográfica do rio Guapi-Macacu, RJ. A coloração apresenta o padrão de cores Munsell dos solos de cada horizonte, conforme descrito em campo. translocação de incremento de argila e presença de cores brunadas a amareladas no horizonte subsuperficial plânico (Bt) e ocorrem preferencialmente em áreas de sopé de elevação, em transição para áreas de planícies aluviais. As ferramentas pedométricas permitiram uma análise comparativa quantitativa entre perfis de solo hidromórficos, com apoio nas características morfológicas, conforme apresentado no dendrograma de dissimilaridade (Figura 6). Tais ferramentas podem ainda ser úteis para auxiliar a definição de séries, ou sequências típicas de classes de solo com base nas variações de grupamentos texturais ou de características do relevo, tempo ou material de origem (topossequências, cronossequências e litossequências, respectivamente). No entanto, mais pesquisas são necessárias para melhor entendimento da variabilidade de propriedades do solo em profundidade.

\section{Conclusões}

1. A aplicação da técnica de fatiamento do perfil permite identificar mudanças contínuas na composição granulométrica em profundidade, por vezes não percebidas com a análise de dados médios por horizonte (conforme coletado em campo) ou em camadas de espessura predefinida, e permite harmonizar o banco de dados de acordo com o Consórcio Mundial para o Mapeamento de Solos (GlobalSoilMap), ainda que o arranjo dos dados em profundidades predefinidas não permita a identificação de variações sutis.

2. De acordo com a composição granulométrica dos perfis caracterizada com as funções de profundidade, a camada superficial dos Gleissolos e Planossolos é geralmente arenosa, com grande variabilidade de silte e argila, para os Gleissolos, e incremento no conteúdo de argila a partir de $60 \mathrm{~cm}$, nos Planossolos.

3. Neossolos Flúvicos apresentam tendência de incremento contínuo na fração areia total, de acordo com a profundidade, e decréscimo nos teores de silte e areia até $1 \mathrm{~m}$ de profundidade.

4. A padronização de esboços em escala e simbologia comum possibilita a comparação das frações granulométricas dos horizontes dos perfis de solos hidromórficos.

5. A análise de similaridade corrobora as funções aplicadas para a descrição dos componentes da textura em profundidade, com menor diferença entre os perfis de Gleissolos e Planossolos, e maior distinção nos perfis dos Neossolos Flúvicos. 


\section{Agradecimentos}

À Coordenação de Aperfeiçoamento de Pessoal de Nível Superior (Capes), ao Conselho Nacional de Desenvolvimento Científico $(\mathrm{CNPq})$ e à Fundação de Amparo à Pesquisa do Estado do Rio de Janeiro (Faperj), pelo apoio financeiro e bolsas.

\section{Referências}

ARROUAYS, D.; MCKENZIE, N.; HEMPEL, J.; FORGES, A.R. de; MCBRATNEY, A.B. (Ed.). GlobalSoilMap: basis of the global spatial soil information system. Boca Raton: CRC Press, 2014. DOI: $10.1201 / \mathrm{b} 16500$.

ARROUAYS, D.; VION, I.; KICIN, J.L. Spatial analysis and modeling of topsoil carbon storage in temperate forest humic loamy soils of France. Soil Science, v.159, p.191-198. 1995. DOI: 10.1097/00010694-199515930-00006.

BEAUDETTE, D.E.; ROUDIER P.; O'GEEN, A.T. Algorithms for quantitative pedology: a toolkit for soil scientists. Computers and Geosciences, v.52, p.258-268, 2013. DOI: 10.1016/j. cageo.2012.10.020.

BISHOP, T.F.A.; MCBRATNEY, A.B.; LASLETT, G.M. Modelling soil attribute depth functions with equal-area quadratic smoothing splines. Geoderma, v.91, p.27-45, 1999. DOI: 10.1016/ S0016-7061(99)00003-8.

CARVALHO JUNIOR, W.; PEREIRA, N.R.; CHAGAS, C.S.; BHERING, S.B.; CALDERANO FILHO, B. Pedologia quantitativa: o perfil mediano e o perfil médio. In: CONGRESSO BRASILEIRO DE CIÊNCIA DO SOLO, 35., 2015, Natal. O solo e suas múltiplas funções: resumos. Natal: Sociedade Brasileira de Ciência do Solo, 2015. 4p.

CLAESSEN, M.E.C. (Org.). Manual de métodos de análise de solo. 2.ed. rev. e atual. Rio de Janeiro: EMBRAPA-CNPS, 1997. 212p. (EMBRAPA-CNPS. Documentos, 1).

FERNANDEZ-ILLESCAS, C.P.; PORPORATO, A.; LAIO, F., RODRIGUEZ-ITURBE, I. The ecohydrological role of soil texture in a water-limited ecosystem. Water Resources Research, v.37, p.2863-2872, 2001. DOI: 10.1029/2000WR000121.

FERRARI, A.L. Evolução tectônica do Gráben da Guanabara. 2001. 412p. Tese (Doutorado) - Instituto de Geociências, Universidade de São Paulo, São Paulo.

FIDALGO, E.C.C.; PEDREIRA, B. da C.C.G.; ABREU, M.B. de; MOURA, I.B. de; GODOY, M.D.P. Uso e cobertura da terra na bacia hidrográfica do rio Guapi-Macacu. Rio de Janeiro: Embrapa Solos, 2008. 31p. (Embrapa Solos. Documentos, 105).

HARTEMINK, A.E.; HEMPEL, J.; LAGACHERIE, P.; MCBRATNEY, A.; MCKENZIE, N.; MACMILLAN, R.A.; MINASNY, B.; MONTANARELLA, L.; SANTOS, M.L. de M.; SANCHEZ, P.; WALSH, M.; ZHANG, G.-L. GlobalSoilMap.net - a new digital soil map of the world. In: BOETTINGER, J.L.; HOWELL, D.W.; MOORE, A.C.; HARTEMINK, A.E.; KIENASTBROWN, S. (Ed.). Digital soil mapping. Wageningen: Springer,
2010. p.423-428. (Progress in soil science, 2). DOI: 10.1007/97890-481-8863-5_33.

JENNY, H. The soil resource: origin and behavior. New York: Springer, 1980. (Ecological studies, 37). DOI: 10.1007/978-14612-6112-4.

KAUFMAN, L.; ROUSSEEUW, P.J. Finding groups in data: an introduction to cluster analysis. Hoboken: J. Wiley, 2005. 368p. (Wiley series in probability and statistics). DOI: 10.1002/9780470316801.

KLINGEBIEL, A.A. Land classification for use in planning. Agriculture yearbook. Washington: Department of Agriculture, 1963. p.399-407. Disponível em: <http://naldc.nal.usda.gov/ download/IND43861772/PDF>. Acesso em: 15 nov. 2015.

KÖPPEN, W.P. Climatologia: con un estudio de los climas de la tierra. Panuco: Fondo de Cultura Economica, 1948. 479p.

LACOSTE, M.; MINASNY, B.; MCBRATNEY, A.B.; MICHOT, D.; VIAUD, V.; WALTER, C. High resolution 3D mapping of soil organic carbon in a heterogeneous agricultural landscape. Geoderma, v.213, p.296-311, 2014. DOI: 10.1016/j. geoderma.2013.07.002.

LIU, F.; ROSSITER, D.G.; SONG, X.-D.; ZHANG, G.-L.; YANG, R.-M.; ZHAO, Y.-G.; LI, D.-C.; JU, B. A similaritybased method for three-dimensional prediction of soil organic matter concentration. Geoderma, v.263, p.254-263, 2016. DOI: 10.1016/j.geoderma.2015.05.013.

MALONE, B.P.; MCBRATNEY, A.B.; MINASNY, B. Empirical estimates of uncertainty for mapping continuous depth functions of soil attributes. Geoderma, v.160, p.614-626, 2011. DOI: 10.1016/j. geoderma.2010.11.013.

MALONE, B.P.; MINASNY, B.; MCBRATNEY, A.B. Mapping continuous soil depth functions in the Edgeroi District, NSW, Australia, using terrain attributes and other environmental factors. In: GEOMORPHOMETRY, 2009, Zurich. Proceeding. Zurich: International Society for Geomorphometry, 2009. p.90-97. Disponível em: <http://www.geomorphometry.org/sites/default/ files/malone2009geomorphometry.pdf > . Acesso em: 15 nov. 2015.

MCBRATNEY, A.B.; ODEH, I.O.A.; BISHOP, T.F.A.; DUNBAR, M.S.; SHATAR, T.M. An overview of pedometric techniques for use in soil survey. Geoderma, v.97, p.293-327, 2000. DOI: 10.1016/S0016-7061(00)00043-4.

MINASNY, B.; MCBRATNEY, A.B. A conditioned Latin hypercube method for sampling in the presence of ancillary information. Computers and Geosciences, v.32, p.1378-1388, 2006. DOI: 10.1016/j.cageo.2005.12.009.

MINASNY, B.; MCBRATNEY A.B.; MENDONÇA-SANTOS, M.L.; ODEH, I.O.A.; GUYON, B. Prediction and digital mapping of soil carbon storage in the Lower Namoi Valley. Australian Journal of Soil Research, v.44, p.233-244, 2006. DOI: 10.1071/ SR05136.

MISHRA, U.; LAL, R.; SLATER, B.; CALHOUN, F.; LIU, D.S.; VAN MEIRVENNE, M. Predicting soil organic carbon stock using profile depth distribution functions and ordinary kriging. Soil Science Society of America Journal, v.73, p.614-621, 2009. DOI: 10.2136/sssaj2007.0410.

Pesq. agropec. bras., Brasília, v.51, n.9, p.1326-1338, set. 2016 DOI: $10.1590 / \mathrm{S} 0100-204 \mathrm{X} 2016000900032$ 
MOORE, I.D.; GESSLER, P.E.; NIELSEN, G.A.; PETERSON, G.A. Soil attribute prediction using terrain analysis. Soil Science Society of America Journal, v.57, p.443-452, 1993. DOI: 10.2136/ sssaj1993.03615995005700020026x.

ODGERS, N.P.; LIBOHOVA, Z.; THOMPSON, J.A. Equalarea spline functions applied to a legacy soil database to create weighted-means maps of soil organic carbon at a continental scale. Geoderma, v.189-190, p.153-163, 2012. DOI: 10.1016/j. geoderma.2012.05.026.

PÁDUA, E.J. de; GUERRA, A.R.; ZINN, Y.L. Modelagem da densidade do solo em profundidade sob vegetação nativa em Minas Gerais. Revista Brasileira de Ciência do Solo, v.39, p.725-736, 2015. DOI: $10.1590 / 01000683$ rbcs20140028.

PEDREIRA, B. da C.C.G.; FIDALGO, E.C.C.; ABREU, M.B. de. Mapeamento do uso e cobertura da terra da bacia hidrográfica do rio Guapi-Macacu, RJ. In: SIMPÓSIO BRASILEIRO DE SENSORIAMENTO REMOTO, 14., 2009, Natal. Anais. Natal: INPE, 2009. p.2111-2118.

PINHEIRO, H.S.K. Métodos de mapeamento digital aplicados na predição de classes e atributos dos solos da Bacia Hidrográfica do Rio Guapi Macacu, RJ. 2015. 178p. Tese (Doutorado) Universidade Federal do Rio de Janeiro, Rio de Janeiro.

PINHEIRO, H.S.K.; ANJOS, L.H.C. dos; CHAGAS, C. da S. Mapeamento digital de solos por redes neurais artificiais: estudo de caso: bacia hidrográfica do Rio Guapi-Macacu, Rio de Janeiro. Saarbrücken: Novas Edições Acadêmicas, 2014. 172p.

PONCE-HERNANDEZ, R.; MARRIOTT, F.H.C.; BECKETT, P.H.T. An improved method for reconstructing a soil profile from analyses of a small number of samples. Journal of Soil Science, v.37, p.455-467, 1986. DOI: 10.1111/j.1365-2389.1986. tb00377.x.

PROJETO MACACU. Planejamento estratégico da região hidrográfica dos Rios Guapi-Macacu e Caceribu-Macacu. Niterói: UFF/FEC, 2010. 544p.

ROUDIER, P.; HEWITT, A.E.; BEAUDETTE, D.E. A conditioned Latin hypercube sampling algorithm incorporating operational constraints. In: MINASNY, B.; MALONE, B.P.; MCBRATNEY, A.B. (ED.). Digital soil assessments and beyond. London: CRC Press, 2012. p.227-232.

SANTOS, H.G. dos; JACOMINE, P.K.T.; ANJOS, L.H.C. dos; OLIVEIRA, V.A. de; LUMBRERAS, J.F.; COELHO, M.R.; ALMEIDA, J.A. de; CUNHA, T.J.F.; OLIVEIRA, J.B. de. Sistema brasileiro de classificação de solos. 3.ed. rev. e ampl. Rio de Janeiro: Embrapa Solos, 2013. 353p.

SANTOS, R.D. dos; LEMOS, R.C. de; SANTOS, H.G. dos; KER, J.C.; ANJOS, L.H.C. dos. Manual de descrição e coleta de solo no campo. 5.ed. rev. e ampl. Viçosa: Sociedade Brasileira de Ciência do Solo; Rio de Janeiro: Embrapa-CNPS, 2005. 92p.

SILVER, W.L.; NEFF, J.; MCGRODDY, M.; VELDKAMP, E.; KELLER, M.; COSME, R. Effects of soil texture on belowground carbon and nutrient storage in a lowland Amazonian forest ecosystem. Ecosystems, v.3, p.193-209, 2000. DOI: 10.1007/ s100210000019.

THE R FOUNDATION. R: The R Project for Statistical Computing. Vienna: The R Foundation, 2013. Disponível em: <www.r-project. org/>. Acesso em: 15 nov. 2015.

THOMPSON, J.A.; ROECKER, S.; GRUNWALD, S.; OWENS, P.R. Digital soil mapping: interactions and applications for hydropedology. In: LIN, H. (Ed.). Hydropedology: synergistic integration of soil science and hydrology. Waltham: Elsevier, 2012. p.665-709. DOI: 10.1016/b978-0-12-386941-8.00021-6.

WEBSTER, R.; OLIVER, M. Statistical methods in soil and land resource survey. Osford: Oxford University, 1990. 316p.

WIESE, L.; ROS, I.; ROZANOV, A.; BOSHOFF, A.; CLERCQ, W. de; SEIFERT, T. An approach to soil carbon accounting and mapping using vertical distribution functions for known soil types. Geoderma, v.263, p.264-273, 2016. DOI: 10.1016/j. geoderma.2015.07.012.

ZINN, Y.L.; LAL, R.; RESCK, D.V.S. Texture and organic carbon relations described by a profile pedotransfer function for Brazilian Cerrado soils. Geoderma, v.127, p.168-173, 2005. DOI: 10.1016/j. geoderma.2005.02.010.

Recebido em 31 de agosto de 2015 e aprovado em 10 de novembro de 2016 